$\begin{array}{cc}\text { ACADEMIA ROMÂNĂ } & \text { Rev. Roum. Chim., } \\ \text { Revo, 65(5), 447-459 } \\ \text { Revue Roumaine de Chimie } & \text { DOI: 10.33224/rrch.2020.65.5.04 } \\ \text { http://web.icf.ro/rrch/ } & \end{array}$

\title{
COMPUTING TOPOLOGICAL INDICES OF CRYSTALLOGRAPHIC STRUCTURES
}

\author{
Muhammad Aamer RASHID, ${ }^{\mathrm{a}, *}$ Sarfraz AHMAD, ${ }^{\mathrm{a}}$ Muhammad Kamran SIDDIQUI ${ }^{\mathrm{a}}$ \\ and Muhammad NAEEM ${ }^{\mathrm{b}}$
}

${ }^{a}$ Department of Mathematics, COMSATS University Islamabad, Lahore Campus, 54000, Pakistan

${ }^{b}$ Department of Mathematics and Statistics, Institute of Southern Punjab, Multan, 66000, Pakistan

Received December 9, 2018

Graph theory plays a vital role in modeling and designing any chemical structure or chemical network. Chemical graph theory helps in understanding about the molecular structural properties of a molecular graph. The molecular graph is a graph consists of atoms called vertices and the chemical bond between atoms called edges. A molecular descriptors (topological index) is a numeric amount related with a graph which describes the topology of the graph and is invariant under graph automorphism. In this article, we study the chemical graphs of Titanium Difluoride $\mathrm{TiF}_{2}$ and Crystallographic Structure of $\mathrm{Cu}_{2} \mathrm{O}$. Moreover, we compute and give closed formulas of degree based additive molecular descriptors (topological indices).

\section{INTRODUCTION}

Graph theory contributes a prominent role in the field of chemical sciences. This theory is proficient for modeling and designing of chemical structures and complex networks. The manipulation and examination of chemical structural information is made conceivable by using molecular descriptors. The chemical graph theory applies graph theory to mathematical modeling of molecular phenomena, which is helpful for the study of molecular structure. Chemical compounds have a variety of applications in chemical graph theory, drug design, etc. A great variety of topological indices are studied and used in theoretical chemistry, pharmaceutical researchers see. $^{1-3}$

A chemical structure can be represented by using graph theory, where vertices denotes atoms and edges denotes molecular bond. A topological index is a numeric number which indicates some useful information about molecular structure. It is the numerical invariants of a molecular graph and are useful to correlate with their bioactivity and physio-chemical properties. Researchers have found topological index to be powerful and useful tool in the description of molecular structure. Some applications related to topological indices of molecular graphs are given in. ${ }^{4,5}$

There are certain chemical compounds that are useful for the survival of living things. Carbon, oxygen, hydrogen and nitrogen are the main elements that helps in the production of cells in the living things. Carbon is an essential element for human life. It is useful in the formation of proteins, carbohydrates and nucleic acids. It is vital for the growth of plants in the form of carbon dioxide. The carbon atoms can bond together in various ways, called allotropes of carbon. The well known forms are graphite and diamond. Recently, many new forms have been discovered including nanotubes, buckminster fullerene and sheets, crystal cubic structure. ${ }^{6,7}$

\footnotetext{
*Corresponding author: aamerrasheed7869@gmail.com
} 
A topological index is a numeric amount related with a graph which describes the topology of the graph and is invariant under graph automorphism. There are some real classes of topological indices, for example, distance based topological indices, degree based topological indices and numbering related polynomials and indices of graphs. The idea of topological indices introduced by Wiener. ${ }^{8}$ The Wiener index is the first and most concentrated topological index, both from theoretical perspective and applications see. ${ }^{9}$

The most seasoned topological index which was presented by I. Gutman and N. Trinajstic is the first Zagreb index, in light of degree of vertices of $\boldsymbol{G}$ in. ${ }^{10}$ Taken after by the first and second Zagreb indices, B. Furtula and I. Gutman ${ }^{11}$ presented Forgotten topological indices which was characterized as:

$$
F(G)=\Sigma_{p q \in F(C ;}\left(\xi_{*}^{2}+\xi_{q}{ }^{2}\right)
$$

In 2015, Gutman et. al. argue that the prescient capacity, acentric factor and entropy of Forgotten Topological index is practically like that of first Zagreb index, and the correlation coefficients between these two is bigger than 0.95. In 2014, Sunet et.al. found some essential type of forgotten topological index and announced that such index can fortify the physico-chemical flexibility of Zagreb indices. Recently, Gao et al. ${ }^{12}$ showed the forgotten topological index of some noteworthy medication atomic structures.

Spurred by the achievement of the ABC index, Furtula et.al., ${ }^{13}$ set forth its changed adaptation and they named it "Augmented Zagreb index" and is characterized as:

$$
A Z I(G)=\Sigma_{p q \in E(G)}\left(\frac{\xi_{p} \times \xi_{q}^{k}}{\xi_{p}+\xi_{m}-2}\right)^{3}
$$

Another topological index based on the vertex degree is the Balaban index. ${ }^{14,15}$ This index for a graph $\boldsymbol{G}$ of order $\boldsymbol{n}$, size $\boldsymbol{m}$ is defined as:

$$
f(G)=\frac{m}{m-n+2} \Sigma_{p q \in E(G)} \frac{1}{\sqrt{\xi_{p} \times \xi_{q}}}
$$

where $\xi_{v}, \xi_{q}$ are the degrees of the vertices $p, q \in V(G)$ The redefined version of the Zagreb indices were defined by Ranjini et al., ${ }^{16}$ namely, the redefined first, second and third Zagreb indices for a graph $G$ as;

$$
\begin{gathered}
\operatorname{ReZ} G_{1}(G)=\Sigma_{p q \in E(G)} \frac{\xi_{p}+\xi_{q}}{\xi_{q} \times \xi_{p}} \\
R e Z G_{2}(G)=\Sigma_{p q E E(G)} \frac{\xi_{p} \times \xi_{q}^{7}}{\xi_{p}+\xi_{q}} \\
R e Z G_{9}(G)=\Sigma_{p q E E(G)}\left(\xi_{p} \times \xi_{q}\right)\left(\xi_{p}+\xi_{q}\right)(6)
\end{gathered}
$$

For further study of topological indices of various graph families. ${ }^{17-25}$

\section{Crystallographic structure of $\mathrm{Cu}_{2} \mathrm{O}$}

Among different transition metal oxides, $\mathrm{C}_{2} \mathrm{u}_{2} \mathrm{O}$ has pulled in extensive consideration as of late attributable to its recognized properties and nontoxic nature, minimal effort, plenitude, and basic creation process. These days, the promising uses of $\mathrm{Cu}_{2} \mathrm{O}$ chiefly concentrate on chemical sensors, solar oriented cells, photocatalysis, lithium-particle batteries and catalysis. The chemical graph of Crystallographic structure of $\mathrm{Cu}_{2} \mathrm{O}$ described in Figure 1 and Figure 2, for more information about this structure see. ${ }^{11-24}$ Let $G \cong C u_{2} O[m, n, t]$ be the chemical graph of $C u_{2} O$ with $m \times n$ unit cells in the plane and $\boldsymbol{t}$ layers. We construct this graph first by taking $m \times n$ unites in the $m \boldsymbol{m}$-plane and then storing it up in $t$ layers. The number of vertices and edges of $c u_{2} 0\left[m_{1} n, t\right]$ are $(m+1)(n+1)(t+1)+5 m n t$ and $8 m m t$, respectively. In $C_{2} u_{2} \boldsymbol{v}\left[\boldsymbol{m}, \boldsymbol{n}_{1} t\right]$ the number zero degree vertices is 4 , the number of one degree vertices is $4 m+4 n+4 t-0$, the number of two degree vertices is $4 m n t+2 m n+2 m t+2 n t-4 n-4 m-4 t+6$ and the number of four degree vertices is $2 n m t-n m-n t-m t+n+m+t-1$.

\section{Main Results for Crystallographic structure of $\mathrm{Cu}_{2} \mathrm{O}$}

In this section, we compute the general result of topological indices for Crystallographic structure of $C u_{2} O$. More preciously, we computed additive topological indices namely Forgotten index, Augmented index, Balaban index and Re-defined Zagreb indices for $C u_{n} O[m, n, t]$. In addition, we give graphical comparison and application of these indices. 
(a)
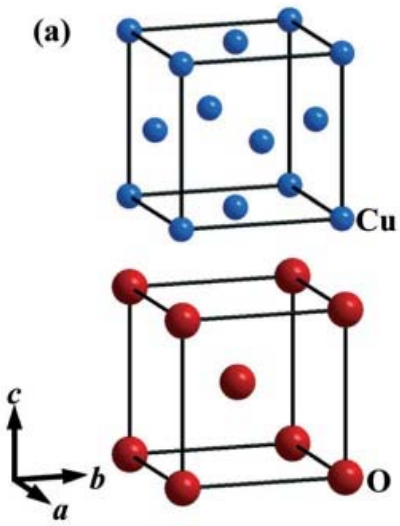

(b)

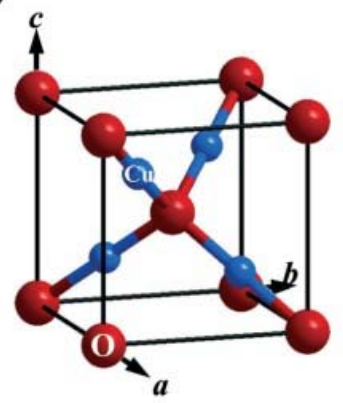

Fig. 1 - Crystallographic structure of $\mathrm{Cu}_{2} \mathrm{O}$. (a) In lattice of $\mathrm{Cu}_{2} \mathrm{O}$ the structural characteristics of the atoms of $\mathrm{Cu}$ and $\mathrm{O}$. The lattice of $\mathrm{Cu}_{2} \mathrm{O}$ is formed by interpenetrating the lattices of $\mathrm{Cu}$ and $\mathrm{O}$ into each other. (b) Unit cell of $\mathrm{Cu} \mathrm{O}_{2} \mathrm{O}$, where copper and oxygen atoms are shown in small blue and in large red spheres."

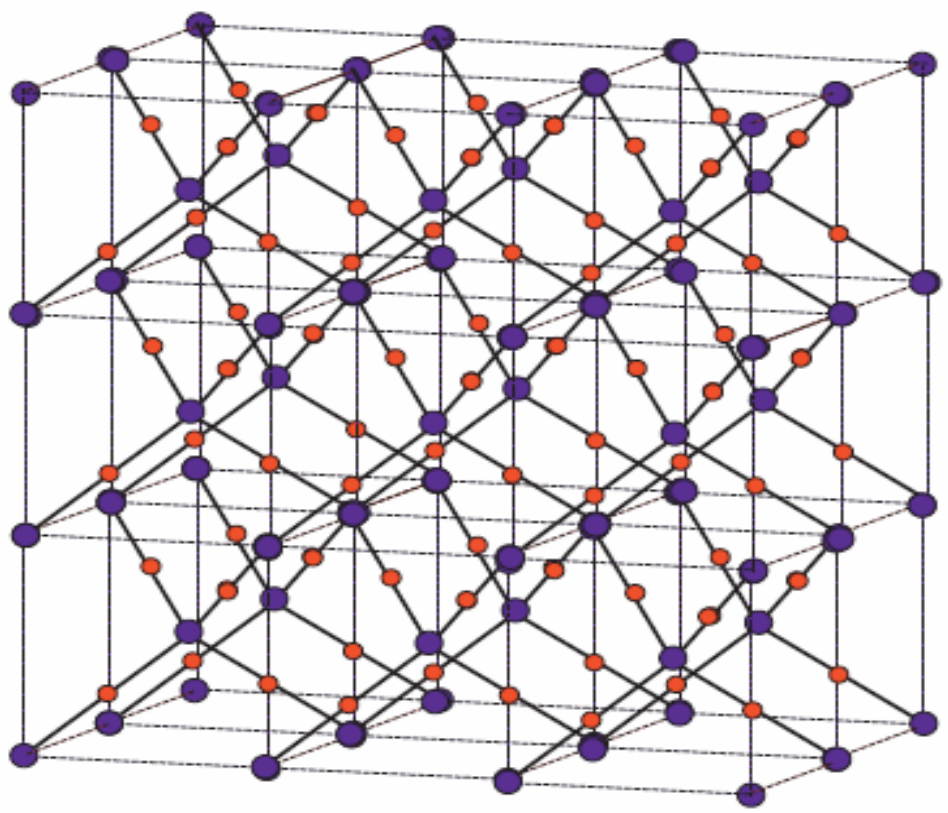

Fig. 2 - Crystallographic structure of $\mathrm{Cu}_{2} \mathrm{O}[3,2,3]$

Table 1

Edge partition of $C u_{2} O[m, n, t]$ based on degrees of end vertices of each edge

\begin{tabular}{c|c|c}
\hline$\left(\xi_{2}, \xi_{a}\right)$ & Frequency & Set of Edges \\
\hline$(1,2)$ & $4 n+4 m+4 t-8$ & $E_{1}$ \\
$(2,2)$ & $4 m m+4 n t+4 m t-8 n-8 m-8 t+12$ & $E_{2}$ \\
$(2,4)$ & $4(2 n m t=n m-n t-m t+n+m+t=1)$ & $E_{3}$ \\
\hline
\end{tabular}

\section{- Forgotten index $\left.F\left(\mathrm{Cu}_{2} O\left[m_{1}, \boldsymbol{n}, t\right]\right]\right)$}

Let $G$ be the graph of carbon graphite $C u, O[m, n, t]$. Now by using equation (1) together with Table 1 we have:

$$
F(G)=\Sigma_{p q \in E(G)}\left(\xi_{p}{ }^{2}+\xi_{q}{ }^{2}\right)
$$




$$
\begin{aligned}
F\left(C u_{2}\right. & O[m, n, t])=\sum_{p q \in S_{i}}\left[\xi_{p}{ }^{2}+\xi_{q}{ }^{2}\right]+\sum_{p q \in S_{s}}\left[\xi_{p}{ }^{2}+\xi_{q}{ }^{2}\right]+\sum_{p q \in E_{p}}\left[\xi_{p}{ }^{2}+\xi_{q}{ }^{2}\right] \\
& =5\left|E_{1}\left(C u_{2} O[m, n, t]\right)\right|+8\left|E_{2}\left(C u_{2} O[m, n, t]\right)\right| \\
& +2 O\left|E_{3}\left(C u_{2} O[m, n, t]\right)\right| \\
& =5(4 n+4 m+4 t-8)+8(4 m m+4 n t+4 m t-8 n-8 m-8 t+12) \\
& +2 O(4(2 n m t-n m-n t-m t+n+m+t-1)) \\
& =36 n+36 m+36 t-24-48 n m-48 n t-48 m t+160 n m t .
\end{aligned}
$$

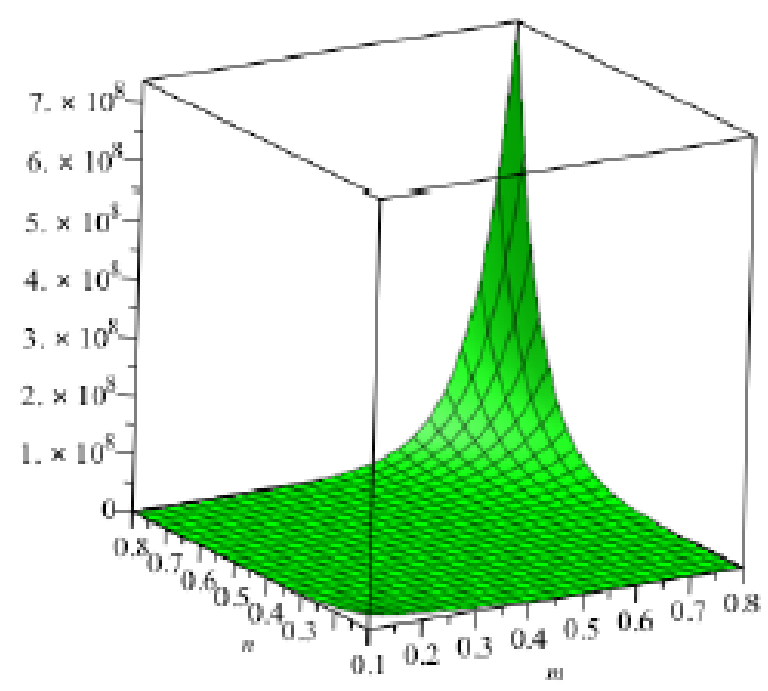

Fig. 3 - The graphical representation of Forgotten index.

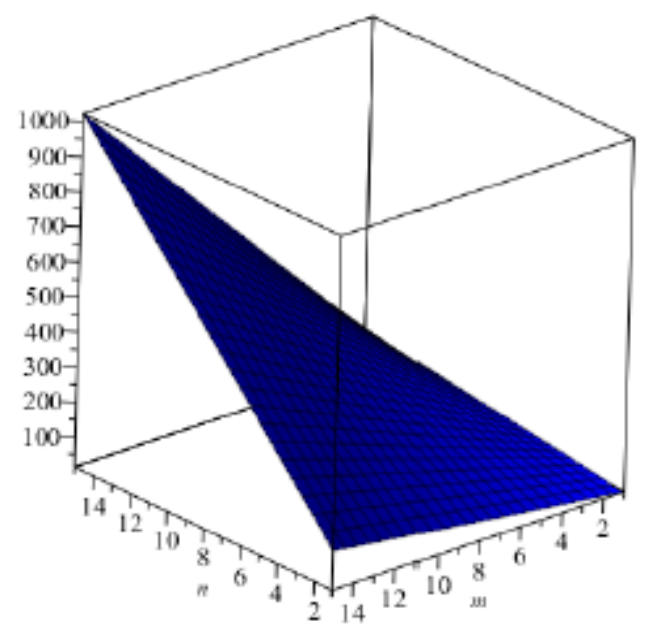

Fig. 4 - The graphical representation of Augmented Zagreb index.

- Augmented Zagreb index $\left.A Z I\left(\mathrm{Cu}_{z} O[\mathrm{~m}, \mathrm{n}, \mathrm{t}]\right]\right)$
Now using equation (2) together with Table 1 we have

Let $G$ be the graph of $G \simeq C \boldsymbol{u}_{2} a[m, \boldsymbol{n}, \mathbf{t}]$.

$$
A Z I(G)=\Sigma_{p q \in E(G)}\left(\frac{\xi_{p} \times \xi_{q}}{\xi_{p}+\xi_{p}-2}\right)^{3}
$$




$$
\begin{aligned}
\left.A Z I\left(C u_{2} O{ }_{-} m, n, t\right]\right)=\sum_{p q \in E_{1}}\left(\frac{\xi_{p} \times \xi_{q}}{\xi_{p}+\xi_{q}-2}\right)^{3}+\sum_{p q \in E_{p}}\left(\frac{\xi_{p} \times \xi_{q}}{\xi_{p}+\xi_{q}-2}\right)^{3}-\sum_{p q \in E_{n}}\left(\frac{\xi_{p} \times \xi_{q}}{\xi_{p}+\xi_{q}-2}\right)^{3} \\
\\
A Z I(G)=8\left|E_{1}\left(C u_{2} O[m, n, t]\right)\right|+8\left|E_{2}\left(C u_{2} \sigma[m, n, t]\right)\right| \\
+8\left|E_{3}\left(C u_{2} O[m, n, t]\right)\right| \\
=8[4 n+4 m+4 t-8)+8(4 n m+4 n t+4 m t-8 n-8 m-8 t+12) \\
+8(4(2 n m t-n m-n t-m t+n+m+t-1)) \\
=64 m m t .
\end{aligned}
$$

\section{- Balaban index $/\left(C u_{2} O[m, n, t]\right]$}

Let $G$ be the graph of $G \cong C^{\prime} u_{2} u[m, n, t]$.
Now using equation (3) together with Table 1 we have:

$$
\begin{aligned}
& I(G)=\frac{m}{m-n+2} \Sigma_{p q \in E(G)} \frac{1}{\sqrt{\xi_{p} \times \xi_{q}}} \\
& J(G)=\frac{m}{m-n+2}\left[\Sigma_{p q \in \Sigma_{1}} \frac{1}{\sqrt{\xi_{p} \times \xi_{q}}}+\Sigma_{p q \in \Sigma_{z}} \frac{1}{\sqrt{\xi_{p} \times \xi_{q}}}\right]+\frac{m}{m-n+2}\left[\Sigma_{p q \in \Sigma_{g}} \frac{1}{\sqrt{\xi_{q} \times \xi_{q}}}\right] \\
& =\frac{9 m n t}{2 m n t-m n-m t-m-n t-n-t+1} \\
& \times\left[\frac{1}{\sqrt{2}}\left|E_{1}\left(C \iota_{2} O[m, n, t]\right)\right|+\frac{1}{2}\left|E_{2}\left(C \iota_{2} O[m, n, t]\right)\right|+\frac{1}{\sqrt{8}}\left|E_{3}\left(C \iota_{2} O[m, n, t]\right)\right|\right] \\
& J(G)=\frac{s_{m n t}}{2 m n t-m-n-m t-m-n t-n-t+1} \\
& \times\left[\frac{1}{\sqrt{2}}(4 n+4 m+4 t-8)+\frac{1}{2}(4 n m+4 n t+4 m t-8 n-8 m-8 t+17)\right] \\
& +\frac{\theta m n t}{2 m n t-m n-m t-m-n t-n-t+1}
\end{aligned}
$$

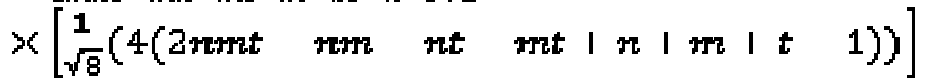

$$
\begin{aligned}
& J(G)=\frac{s_{m n t}}{2 m n t-m n-m t-m-n t-n-t+1} \times\left[\frac{\sqrt{2}}{2}(4 n+4 m+4 t-8)\right]
\end{aligned}
$$

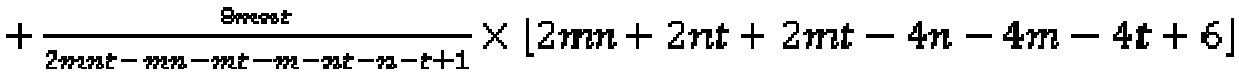

$$
\begin{aligned}
& +\frac{\text { Gmut }}{2 m n t-m n-m t-m-n t-n-t+1} \\
& \times\left[\frac{\sqrt{2}}{2}(2 n m t-n m-n t-m t+n+m+t-1)\right]
\end{aligned}
$$

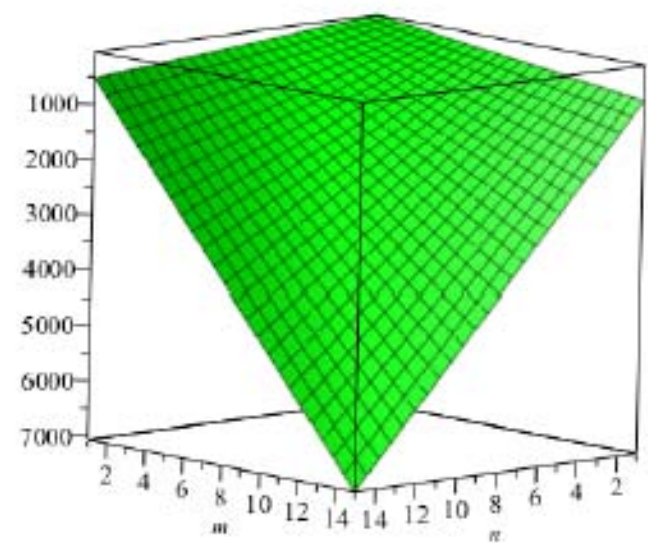

Fig. 5 - The graphical representation of Balaban index. 
- The Redefine Zagreb indices for $C u_{2} O[m, n, t]$
Let $\mathrm{G}$ be the graph of $\boldsymbol{G} \simeq \mathrm{cu}_{z} a[m, n, t]$. Now using equations [(4)-(6)] and Table 1 we have:

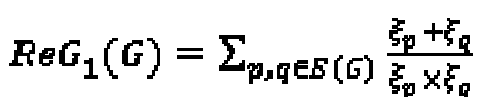

$$
\begin{aligned}
& \operatorname{ReG} G_{1}\left(C u_{2} O[m, n, t]\right)=\Sigma_{p q \in E_{1}} \frac{\xi_{p}+\xi_{q}}{\xi_{p} \times \xi_{p}}+\Sigma_{p q \in \Sigma_{2}} \frac{\xi_{p}+\xi_{q}}{\xi_{p} \times \xi_{q}}+\Sigma_{p q \in \Sigma_{g}} \frac{\xi_{p}+\xi_{q}}{\xi_{p} \times \xi_{q}} \\
& \operatorname{ReG}_{1}\left(C u_{2} O[m, n, t]\right)=\frac{\dot{d}}{2}\left|E_{1}\left(C u_{2} O[m, n, t]\right)\right|+1\left|E_{2}\left(C u_{2} O[m, n, t]\right)\right| \\
& +\frac{3}{4}\left|E_{3}\left(C u_{2} O[m, n, t]\right)\right| \\
& =\frac{3}{2}(4 n+4 m+4 t-8) \\
& +(4 n m+4 n t+4 m t-8 n-8 m-8 t+12) \\
& +\frac{a}{4}(4(2 n m t-n m-n t-m t+n+m+t-1)) \\
& =n+m+t-3+n m+n t+m t+6 n m t \\
& \operatorname{ReG}_{2}(G)=\Sigma_{p, q \in E(G)} \frac{\xi_{p} \times \xi_{q}}{\xi_{p}+\xi_{q}} \\
& R e G_{2}\left(C u_{2} O[m, n, t]\right)=\Sigma_{p q \in E_{1}} \frac{\xi_{p} \times \xi_{q}}{\xi_{p}+\xi_{q}}+\Sigma_{p q \in \Sigma_{2}} \frac{\xi_{p} \times \xi_{q}}{\xi_{p}+\xi_{q}}+\Sigma_{p q \in E_{a}} \frac{\xi_{p} \times \xi_{q}}{\xi_{p}+\xi_{q}} \\
& =\frac{2}{3}\left|E_{1}\left(C u_{2} O[m, n, t]\right)\right|+1\left|E_{2}\left(C u_{2} O[m, n, t]\right)\right| \\
& +\frac{4}{3}\left|E_{3}\left(C u_{2} O[m, n, t]\right)\right| \\
& =\frac{2}{3}(4 n+4 m+4 t-8) \\
& +(4 n m+4 n t+4 m t-8 n-8 m-8 t+12) \\
& +\frac{4}{3}(4(2 n m t-n m-n t-m t+n+m+t-1)) \\
& =\frac{4}{3}-\frac{4}{3} n m-\frac{4}{3} n t-\frac{4}{3} m t+\frac{32}{3} n m t
\end{aligned}
$$

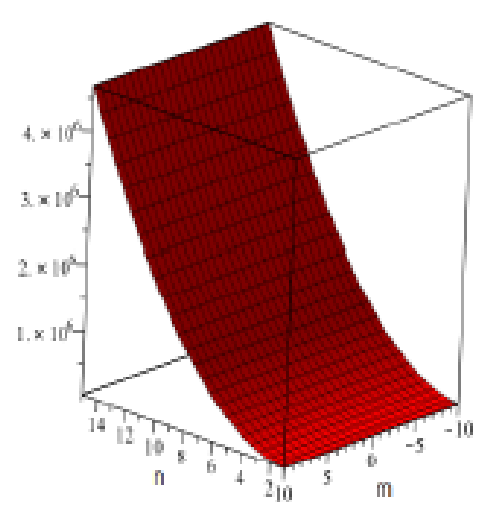

(a)

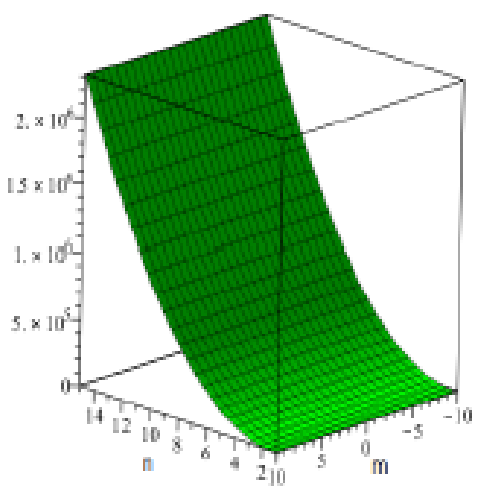

[b]

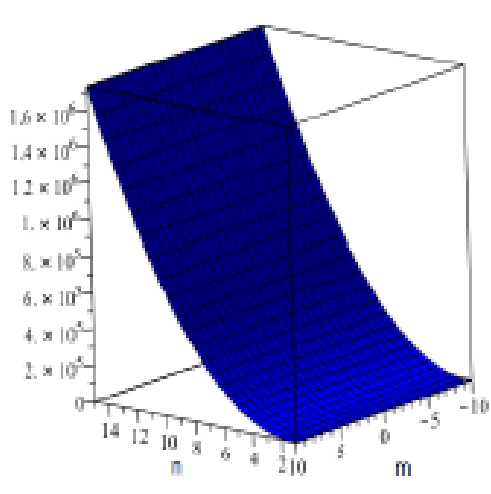

(c)

Fig. 6 - The graphical representation of (a) $\boldsymbol{R e} G_{1}(G)$ index (b) $R e G_{2}(G)$ index (c) $\operatorname{Re} G_{3}(G)$ index.

$$
\begin{aligned}
& \operatorname{ReG}_{3}(G)=\Sigma_{p G \in E(G)}\left(\xi_{p} \times \xi_{q}\right)\left(\xi_{p}+\xi_{q}\right) \\
& R e G_{3}\left(C u_{2} O[m, n, t]\right)=\sum_{p q \in S_{1}}\left(\xi_{p} \times \xi_{q}\right)\left(\xi_{p}+\xi_{q}\right)+\sum_{p q \in E_{p}}\left(\xi_{p} \times \xi_{q}\right)\left(\xi_{p}+\xi_{q}\right) \\
& +\Sigma_{p q \in E_{q}}\left(\xi_{p} \times \xi_{q}\right)\left(\xi_{p}+\xi_{q}\right) \\
& =(2)(3)\left|E_{1}\left(C u_{2} O[m, n, t]\right)\right|+(4)(4)\left|E_{2}\left(C u_{2} Q[m, n, t]\right)\right|
\end{aligned}
$$




$$
\begin{aligned}
& \left.+(3)(6) \mid E_{3}\left(C u_{2} \sigma_{m} m, t\right]\right) \mid \\
& =(2)(3)(4 n+4 m+4 t-8) \\
& +(4)(4)(4 n m+4 n t+4 m t-8 n-8 m-8 t+12) \\
& +(8)(6)(4(2 m m t-n m-n t-m t+n+m+t-1)) \\
& =88 n+88 m+88 t-48-128 n m-128 n t-128 m t+384 n m t
\end{aligned}
$$

\section{Crystal Structure of Titanium Difluoride}

Titanium Difluoride is a water insoluble Titanium source for use in oxygen-sensitive applications, for example, metal production. Fluoride compounds have various applications in current advances and science, from oil refining and drawing to engineered organic chemistry and the making of pharmaceuticals.

The chemical graph of crystal structure of titanium difluoride $T \dot{i} F_{2}[m, n, t]$ is described in Figure 7, for more details see. ${ }^{25,26}$ Let $G \cong T i F_{2}[m, n, t]$ be the chemical graph of $T \dot{L} F_{2}$ with $m \times n$ unit cells in the plane and $t$ layers.
We construct this graph first by taking $\boldsymbol{m} \times \boldsymbol{n}$ unites in the $\boldsymbol{m} n$-plane and then storing it up in $\boldsymbol{t}$ layers. The cardinality of vertices and edges of $T i F_{2}[m, n, t]$ are $12 m n t+2 m n+2 m t+$ $+2 n t+m+n+t+1$ and $32 m n t$, respectively. In $T i F_{2}[m, n, t]$ the number of one degree vertices is 8 , the number of two degree vertices is $4 m+4 n+4 t-12$, the number of four degree vertices is $8 m n t+4 m n+4 m t+4 n t-4 n-$ $-4 m-4 t+6$ and the number of eight degree vertices is $4 m n^{2}-2(m n+m t+n t)+m+$ $+n+t-1$.

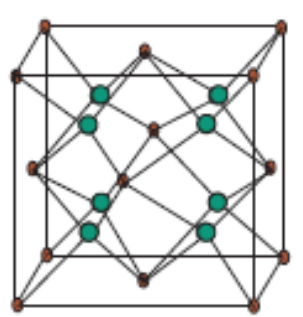

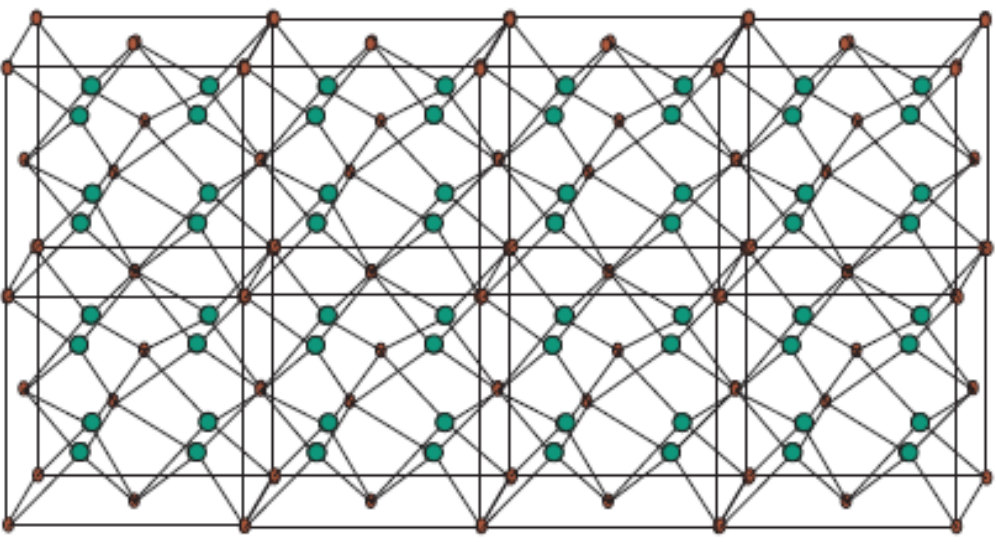

(b)

Fig. 7 - Crystal Structure Titanium Difluoride $\boldsymbol{T} \boldsymbol{i} \boldsymbol{F}_{2}\left[\boldsymbol{m}_{\boldsymbol{1}}, \boldsymbol{n}, \boldsymbol{t}\right]$, (a) represents unit cell of of $\boldsymbol{T} \boldsymbol{i} \boldsymbol{F}_{\mathbf{2}}[\boldsymbol{m}, \boldsymbol{n}, \boldsymbol{t}]$ with $\boldsymbol{T} \dot{\boldsymbol{t}}$ atoms in red and $\boldsymbol{F}$ atoms in green (b) crystal structure of $T \dot{i} \boldsymbol{F}_{2}[4,1,2]$.

Table 2

Edge partition of $\mathbf{T} \boldsymbol{i} \boldsymbol{F}_{\mathbf{2}}[\boldsymbol{m}, \boldsymbol{n}, \boldsymbol{t}]$ based on degrees of end vertices of each edge

\begin{tabular}{c|c|c}
\hline$\left(\xi_{\eta}, \xi_{a}\right)$ & Frequency & Set of Edges \\
\hline$(1,4)$ & 8 & $E_{1}$ \\
$(2,4)$ & $8(m+n+t-3)$ & $E_{2}$ \\
$(4,4)$ & $16(m n+m t+n t)-16(m+n+t)+24$ & $E_{3}$ \\
$(4,8)$ & $32 m n t-16(m t+m n+n t)+8(m+n+t)-8$ & $E_{4}$ \\
\hline
\end{tabular}

Main Results for Crystallographic structure of

\section{$\mathbf{T i F}_{\mathbf{z}}[\mathrm{m}, \mathbf{n}, \boldsymbol{t}]$}

In this section, we compute the general result of topological indices for Crystallographic structure of $T \dot{t} F_{2}[m, n, t]$. More preciously, we computed additive topological indices namely Forgotten index, Augmented index, Balaban index and Redefined Zagreb indices for $T \dot{t} F_{2}[m, n, t]$. In addition, we give graphical comparison and application of these indices. 
- Forgotten index $F\left(\operatorname{TiF}_{z}[\boldsymbol{m}, \boldsymbol{r}, t]\right)$ using equation (1) together with Table 2 we have

Let $G$ be the graph of $G \simeq T \dot{t} F_{2}[m, n, t]$. Now

$$
\begin{aligned}
& F(G)=\sum_{p, q \in E(G)}\left(\xi_{p}^{2}+\xi_{q}^{2}\right) \\
& F\left(T i F_{2}[m, n, t]\right)=\Sigma_{p q \in \Sigma_{1}}\left(\xi_{p}{ }^{2}+\xi_{q}{ }^{2}\right)+\Sigma_{p q \in \Sigma_{q}}\left(\xi_{p}{ }^{2}+\xi_{q}{ }^{2}\right) \\
& +\Sigma_{p q E E_{n}}\left(\xi_{p}{ }^{2}+\xi_{q}{ }^{2}\right)+\Sigma_{p q \in E_{A}}\left(\xi_{p}{ }^{2}+\xi_{q}{ }^{2}\right) \\
& =17\left|E_{1}\left(T t F_{2}[m, n, t]\right)\right|+20\left|E_{2}\left(T t F_{2}[m, n, t]\right)\right| \\
& +32\left|E_{9}\left(T i F_{2}[m, n, t]\right)\right|+80\left|E_{4}\left(T i F_{2}[m, n, t]\right)\right| \\
& F(G)=17(8)+20(8(m+n+t-3))+32(16(m n+m t+n t) \\
& -16(\mathrm{~m}+\mathrm{n}+\boldsymbol{t})+24) \\
& +80(32 m n t-16(m t+m n+n t)+8(m+n+t)-8) \\
& =-216+288 m+288 n+288 t-768 m n-768 m t-768 n t+2560 m n t
\end{aligned}
$$

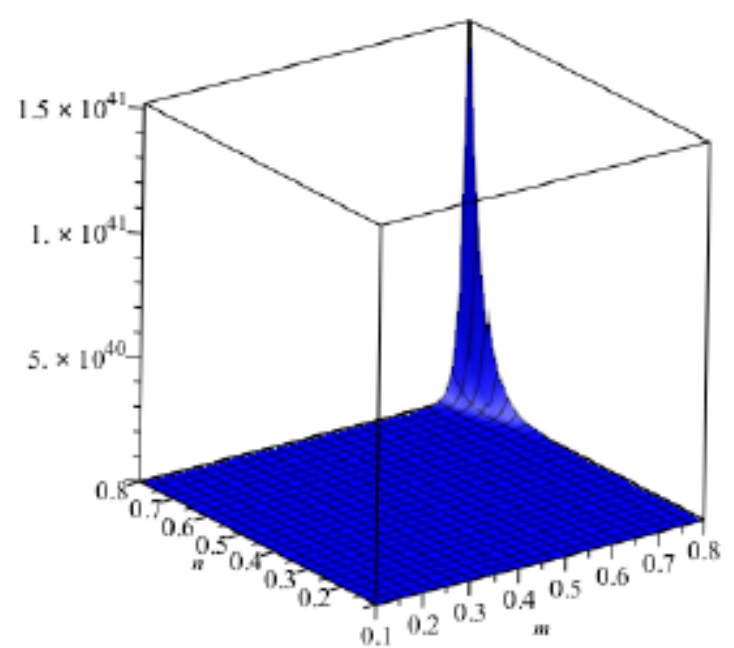

Fig. 8 - The graphical representation of Forgotten index.

$$
\begin{aligned}
& \text { - Augmented Zagreb index } \\
& \operatorname{AZI}\left(\operatorname{TiF}_{2}[\mathrm{~m}, \mathrm{n}, t]\right) \\
& \text { Let } \mathrm{G} \text { be the graph of } \boldsymbol{G} \simeq T \dot{\mathbf{i}} \boldsymbol{F}_{\mathbf{z}}[\boldsymbol{m}, \boldsymbol{r}, \boldsymbol{t}] \text {. By } \\
& A Z r\left(G j=\Sigma_{p, q \in E(G)}\left(\frac{\xi_{p} \times \xi_{p}}{\xi_{p}+\xi_{p}-2}\right)^{3}\right. \\
& A Z I\left(T i F_{2}[m, n, t]\right)=\sum_{p q \in E_{1}}\left(\frac{\xi_{p} \times \xi_{q}}{\xi_{p}+\xi_{q}-2}\right)^{\mathrm{a}}+\sum_{p q \in E_{2}}\left(\frac{\xi_{p} \times \xi_{q}}{\xi_{p}+\xi_{q}-2}\right)^{\mathrm{a}} \\
& +\Sigma_{p q \in \Sigma_{g}}\left(\frac{\xi_{p} \times \xi_{q}}{\xi_{p}+\xi_{q}-2}\right)^{3}+\Sigma_{p q \in \Sigma_{4}}\left(\frac{\xi_{p} \times \xi_{q}}{\xi_{p}+\xi_{q}-2}\right)^{3} \\
& =\frac{64}{27}\left|E_{1}\left(T i F_{2}^{\prime}[m, n, t]\right)\right|+8\left|E_{2}\left(T^{\prime} i F_{2}^{\prime}[m, n, t]\right)\right| \\
& +\frac{E_{12}}{27}\left|E_{3}\left(T i F_{2}[m, n, t]\right)\right| \\
& +\frac{1096}{125}\left|E_{4}\left(\operatorname{TiF}_{2}[m, n, t]\right)\right| \\
& =\frac{64}{27}(8)+8(8(m+n+t-3)) \\
& +\frac{512}{27}(16(m n+m t+n t)-16(m+n+t)+24) \\
& +\frac{40 y_{6}}{125}(32 m n t-16(m t+m n+n t)+8(m+n+t)-8)
\end{aligned}
$$$$
\text { using Table } 2 \text { and equation (2), we have }
$$ 


$$
\begin{aligned}
& =\frac{67264}{3375}+\frac{76736}{3375} \mathrm{mz}+\frac{76736}{3375} \mathrm{n}+\frac{76736}{3375} t-\frac{745472}{3375} \mathrm{mzh}-\frac{745472}{3375} \mathrm{mt} \\
& -\frac{745472}{3375} \mathrm{nt}+\frac{131072}{125} \mathrm{nunt}
\end{aligned}
$$

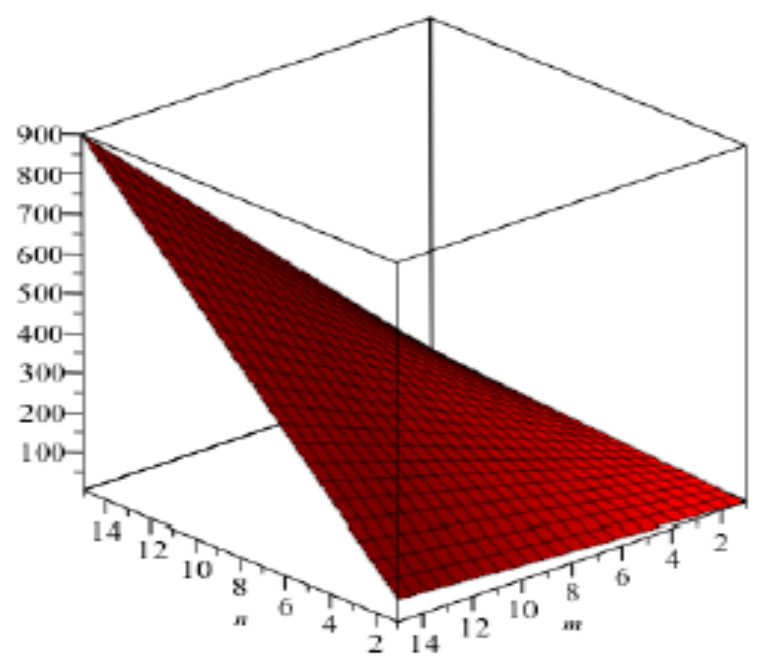

Fig. 9 - The graphical representation of Augmented Zagreb index.

\section{- Balaban index $I\left(\mathbf{T i F}_{\mathbf{z}}[\boldsymbol{m}, \boldsymbol{n}, \boldsymbol{t}]\right)$}

by using Table 2 and equations (3), we have:

Let $G$ be the graph of $G \cong T i F_{2}[m, n, t]$. Now

$$
\begin{aligned}
& I(G)=\frac{m}{m-n+2} \Sigma_{p, q \in E(G)} \frac{1}{\sqrt{\xi_{p} \times \xi_{q}}} \\
& J\left(T i F_{2}[m, n, t]\right)=\frac{m}{m-m+1} \times\left[\Sigma_{p q \in \Sigma_{1}} \frac{1}{\sqrt{\xi_{p} \times \xi_{q}}}+\Sigma_{p q \in \Sigma_{z}} \frac{1}{\sqrt{\xi_{p} \times \xi_{q}}}\right] \\
& +\frac{m}{m-n+2} \times\left[\sum_{p q \in \Sigma_{s}} \frac{1}{\sqrt{\xi_{p} \times \xi_{q}}}+\sum_{p q \in \Sigma_{4}} \frac{1}{\sqrt{\xi_{p} \times \xi_{q}}}\right] \\
& =\frac{32 m n t}{20 m n t-2 m n-2 m t-2 n t-m-n-t+1} \times \\
& \times\left[\frac{1}{2}\left|E_{1}\left(T i F_{2}[m, n, t]\right)\right|+\frac{1}{2 \sqrt{2}}\left|E_{2}\left(T i F_{2}[m, n, t]\right)\right|\right] \\
& +\left[\frac{32 m n t}{2 u m m t-2 m m-2 m t-2 n t-m-n-t+1}\right] \\
& \times\left[\frac{1}{4}\left|E_{3}\left(T t F_{2}[m, n, t]\right)\right|+\frac{1}{4 \sqrt{2}}\left|E_{4}\left(T t F_{2}[m, n, t]\right)\right|\right] \\
& -\frac{32 m n !}{20 m n t-2 m n-2 m t-2 n t-m-n-t+1} \\
& \times\left[\frac{1}{2}(8)+\frac{1}{2 \sqrt{2}}(8(m+n+t-3))\right] \\
& +\left[\frac{1}{4}(16(n m+m t+n t)-16(m+n+t)+24)\right] \\
& +\left[\frac{32 m x t}{20 m m t-2 m n-2 m t-2 n t-m-n-t+1}\right] \\
& \times\left[\frac{1}{4 \sqrt{2}}(32 m n t-16(m t+m n+n t)+8(m+n+t)-8)\right] \\
& =\left[\frac{32 m n t}{20 m n t-2 m n-2 m t-2 n t-m-n-t+1}\right] \times \\
& \times[10+2(m+n+t-3) \sqrt{2}+4 m n+4 m t+4 n t-4 m-4 n-4 t]
\end{aligned}
$$



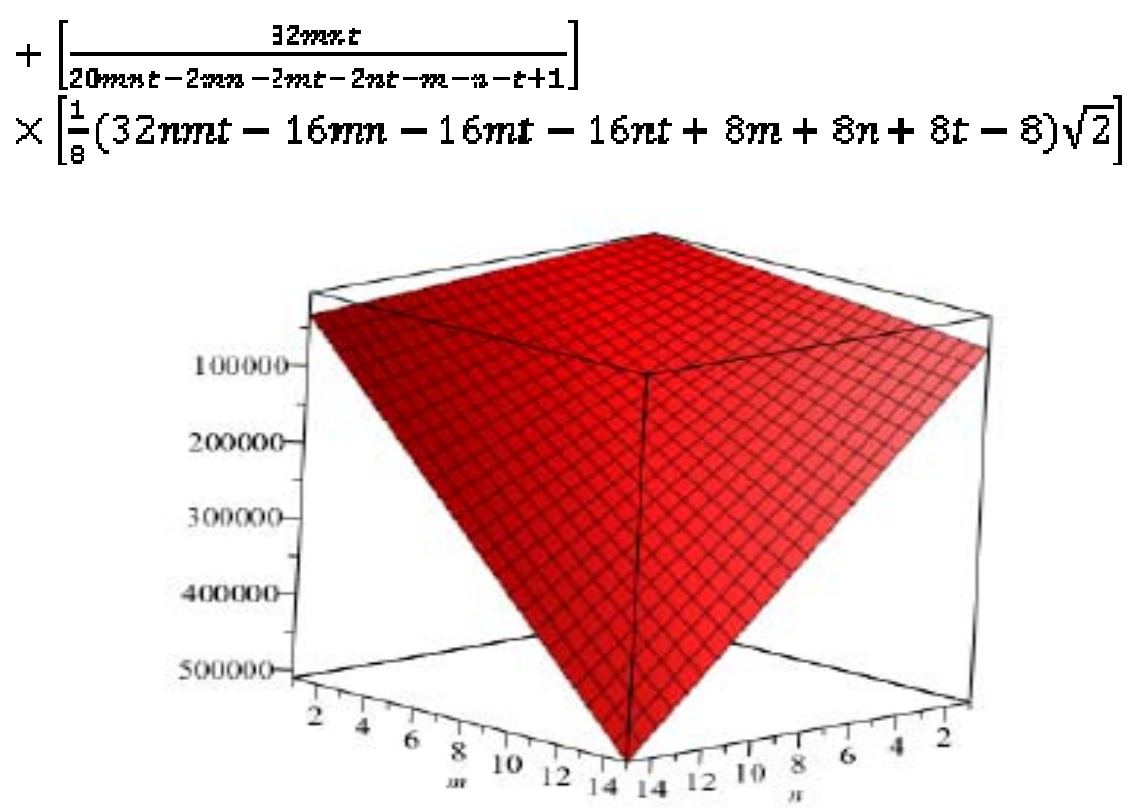

Fig. 10 - The graphical representation of Balaban index.

- The Redefine Zagreb indices of $\mathbf{T i F}_{2}[\boldsymbol{m}, \mathbf{n}, \boldsymbol{t}]$

$$
\begin{aligned}
& \operatorname{ReG}(G)=\Sigma_{p, q \in E(G)} \frac{\xi_{p}+\xi_{q}}{\xi_{p} \times \xi_{q}}
\end{aligned}
$$

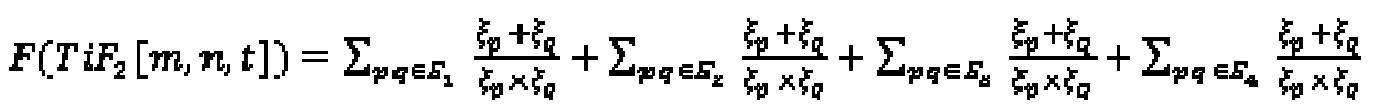

$$
\begin{aligned}
& -\frac{5}{4}\left|E_{1}\left(T i F_{2}[m, n, t]\right)\right|+\frac{3}{4}\left|E_{2}\left(T i F_{2}[m, n, t]\right)\right| \\
& +\frac{1}{2}\left|B_{3}\left(T i F_{2}[m, n, t]\right)\right|+\frac{3}{8}\left|B_{4}\left(T i F_{2}[m, n, t]\right)\right| \\
& =\frac{5}{4}(0)+\frac{3}{4}(0(m+n+t-3))+\frac{1}{2}(16(m n+m t+n t) \\
& -16(m+n+1)+24) \\
& +\frac{3}{8}(32 m n t-16(m t+m n+n t)+8(m+n+t)-8) \\
& =1+m+n+t+2 m n+2 m t+2 n t+12 m n t \\
& \operatorname{ReG}_{2}(G)=\sum_{p, q \in E(G)} \frac{\xi_{p} \times \xi_{q}}{\xi_{p}+\xi_{q}} \\
& R e G_{2}\left(T \dot{t} F_{2}[m, n, t]\right)=\sum_{p q \in \Sigma_{1}} \frac{\xi_{p} \times \xi_{q}}{\xi_{p}+\xi_{q}}+\sum_{p q \in \Sigma_{q}} \frac{\xi_{p} \times \xi_{q}}{\xi_{p}+\xi_{q}}+\sum \frac{\xi_{p} \times \xi_{q}}{\xi_{p}+\xi_{q}}+\sum_{p q \in \Sigma_{\alpha}} \frac{\xi_{p} \times \xi_{q}}{\xi_{p}+\xi_{q}} \\
& =\frac{4}{5}\left|E_{1}\left(T i F_{2}[m, n, t]\right)\right|+\frac{4}{3}\left|E_{2}\left(T i F_{2}[m, n, t]\right)\right| \\
& +2\left|E_{3}\left(T i F_{z}[m, n, t]\right)\right|+\frac{8}{3}\left|E_{4}\left(T i F_{2}[m, n, t]\right)\right| \\
& -\frac{4}{5}(8)+\frac{4}{3}(8(m+n+t-3))+2(16(m n+m t+n t) \\
& -16(m+n+t)+24) \\
& +\frac{8}{3}(32 m n t-16(m t+m n+n t)+8(m+n+t)-8) \\
& =\frac{16}{15}-\frac{32}{3} m n-\frac{32}{3} m t-\frac{32}{3} n t+\frac{256}{3} m n t \\
& \operatorname{ReG}_{3}(G)=\Sigma_{p, q \in E(G)}\left(\xi_{p} \times \xi_{q}\right)\left(\xi_{p}+\xi_{q}\right)
\end{aligned}
$$




$$
\begin{aligned}
& \operatorname{Re} G_{3}\left(T \pm F_{2}[m, n, t]\right. \\
& =\sum_{s t \in E_{1}}\left(\xi_{p} \times \xi_{q}\right)\left(\xi_{p}+\xi_{q}\right)+\sum_{s t \in E_{z}}\left(\xi_{p} \times \xi_{q}\right)\left(\xi_{p}+\xi_{q}\right) \\
& +\sum_{p q \in \Sigma_{\mathrm{q}}}\left(\xi_{p} \times \xi_{q}\right)\left(\xi_{p}+\xi_{q}\right) \\
& +\sum_{p q \in \Sigma_{\alpha}} \xi_{q} \cdot \xi_{q}\left(\xi_{p}+\xi_{q}\right) \\
& =4(5)\left|E_{1}\left(T i F_{2}[m, n, t]\right)\right|+6(8)\left|E_{2}\left(T i F_{2}[m, n, t]\right)\right| \\
& +8(16)\left|E_{3}\left(T i F_{2}[m, n, t]\right)\right|+12(32)\left|E_{4}\left(T i F_{2}[m, n, t]\right)\right| \\
& =20(8)+48(8(m+n+t-3))+128(16(n m+m t+n t) \\
& -16(m+n+t)+24) \\
& +384(32 m n t-16(m t+m n+n t)+8(m+n+t)-8) \\
& =-992+1408 m+1408 n+1408 t-4096 m n-4096 m t \\
& -4096 n t+12288 m n t
\end{aligned}
$$

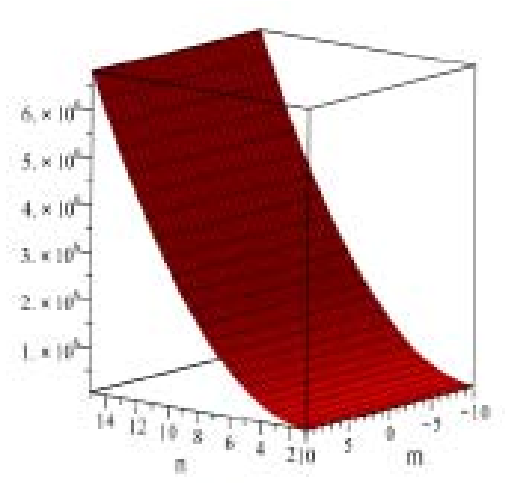

(a)

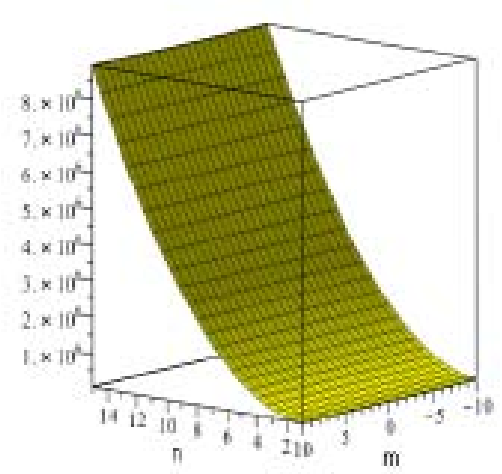

(b)

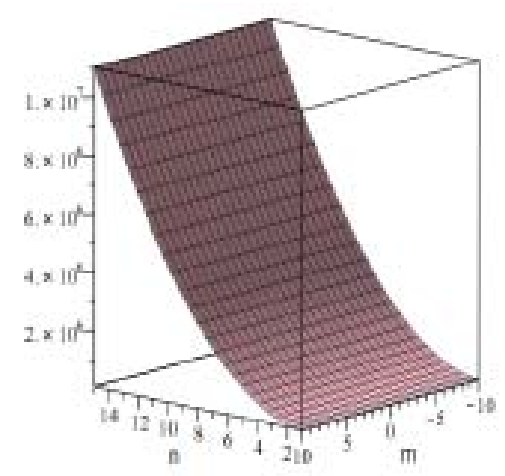

(1)

Fig. 11 - The graphical representation of (a) $\boldsymbol{R e} G_{1}(G)$ index (b) $R \boldsymbol{g} G_{2}[G)$ index (c) $\boldsymbol{R} e G_{3}(G)$ index.

\section{COMPARISONS AND DISCUSSION}

- For the comparison of these indices numerically for $C u_{2} v$, we computed all indices for different values of $\boldsymbol{m}, \boldsymbol{n}, \boldsymbol{t}$. Now, from Table 3 , we can easily see that all indices are in increasing order as the values of $m, n, t$ are increasing. The graphical representations of topological indices for $C u_{2} O[m, n, t]$ are depicted for Forgotten index in Figure 3. The Augmented index in Figure 4, Balaban index in Figure 5 and the redefined Zagreb indices in Figure 6 for certain values of $m, r, t$

Table 3

Numerical computation of all indices for $\mathrm{Cu}_{2} \mathrm{O}$

\begin{tabular}{l|l|l|l|l|c|c}
\hline$[m, n, t]$ & $F(G)$ & $\Lambda Z I(G)$ & $J(G)$ & $R a Z_{1}(G)$ & $R a Z_{2}(G)$ & $\operatorname{ReZ}_{3}(G)$ \\
\hline$[1,1,1]$ & 1812 & 2094 & $3.2 \times 10^{4}$ & 101 & $4.5 \times 10^{6}$ & 212 \\
{$[2,2,2]$} & 5176 & 6344 & $5.3 \times 10^{6}$ & 235 & $12.7 \times 10^{8}$ & 379 \\
{$[3,3,3]$} & 10268 & 12898 & $9.4 \times 10^{8}$ & 330 & $15.3 \times 10^{10}$ & 546 \\
{$[4,4,4]$} & 17788 & 21756 & $14.3 \times 10^{12}$ & 424 & $19.4 \times 10^{16}$ & 713 \\
\hline
\end{tabular}


Table 4

\begin{tabular}{c|c|c|c|c|c|c}
\multicolumn{7}{c}{ Numerical computation of all indices for $T i F_{2}\left[m, n, t^{*}\right.$} \\
\hline$[m, n, t]$ & $F(G)$ & $A Z I(G)$ & $J(G)$ & $\operatorname{Re} Z_{1}(G)$ & $\operatorname{Re} Z_{2}(G)$ & $\operatorname{Ae} Z_{3}(G)$ \\
\hline$[1,1,1]$ & 1812 & 2094 & $4.3 \times 10^{6}$ & 118 & $5.5 \times 10^{7}$ & 314 \\
{$[2,2,2]$} & 6512 & 7354 & $7.3 \times 10^{9}$ & 468 & $15.7 \times 10^{11}$ & 478 \\
{$[3,3,3]$} & 14258 & $148 / 8$ & $10.5 \times 10^{12}$ & $92 /$ & $19.4 \times 10^{15}$ & 646 \\
{$[4,4,4]$} & 24758 & 32766 & $19.2 \times 10^{16}$ & 1537 & $24.5 \times 10^{19}$ & 917 \\
\hline
\end{tabular}

- For the comparison of these indices numerically for $T \dot{t} F_{2}[\boldsymbol{m}, \boldsymbol{n}, t]$, we computed all indices for different values of $\boldsymbol{m}, \boldsymbol{n}, \boldsymbol{t}$. Now, from Table 4, we can easily see that all indices are in increasing order as the values of $\boldsymbol{m}, \boldsymbol{n}, \boldsymbol{t}$ are increasing. The graphical representations of topological indices for $\mathrm{TtF}_{2}[\boldsymbol{m}, \mathrm{r}, \mathrm{t}]$ are depicted for Forgotten index in Figure 8. The Augmented index in Figure 9, Balaban index in Figure 10 and the redefined Zagreb indices in Figure 11 for certain values of $\boldsymbol{m}, \boldsymbol{n}$.

- The forgotten topological index is helpful for testing the substance and pharmacological properties of drug nuclear structures. So in the case of $C u_{2} O$ and $T t F_{2}\left[m, n, t_{-}^{-}\right.$, its increasing value is useful for quick action during chemical reaction for drugs.

The augmented Zagreb index displays a good correlation with the formation heat of heptanes and octane. So our computation for AZI index is play an important rule for formation heat of heptanes and octane as its values are in increasing order.

The Balaban index shows good correlation with entropy of an octane isomers. The computation of Balaban index for $C u_{2} \boldsymbol{O}$ and $T \dot{T} F_{2}[m, n, t]$ provide a positive input for the correlation with entropy of all octane isomers.

The Zagreb types indices and polynomials were found to occur for the computation of the total $\pi$ electron energy of molecules, thus, the total $\pi$ electron energy in increasing order in the case of $C u_{2} O$ and $T i F_{2}[m, n, t]$, for higher values of $m, n, t$.

\section{CONCLUSIONS}

In this paper, we have studied and computed some degree based topological indices for the chemical graph of the crystal structure of titanium difluoride $T \dot{H} F_{2}$ and crystallographic structure of cuprite $\mathbf{C} u_{2} \boldsymbol{O}$. The exact results have been computed for additive topological indices namely Forgotten index, Augmented index, Balaban index and Re-defined Zagreb indices for $C u_{2} O[m, n, t]$ and $T \dot{t} F_{2}[m, n, t]$ for some values of $m, n, t$.

In future we are interested in computing the distance based and counting related topological indices for these structures.

\section{REFERENCES}

1. J.-B. Liu, C. Wang, S. Wang and B. Wei, Bull. Malaysian Mathem. Sci. Soc.,2019, 42, 67-78.

2. J.-B. Liu, J. Zhao and Z. X. Zhu, Int. J. Quantum Chem., 2019, 119, 25-31.

3. J.-B Liu, J. Zhao, J. Min and J. D. Cao, Nature and Society, 2019, 5, 15-25.

4. J.-B. Liu, J. Zhao and Z. Cai, Physica A, 2020, 540, 12-33.

5. J.-B. Liu, J. Zhao, H. H and, Z. Shao, J. Statistical Phys., 2019, 177, 1131-1147.

6. A. Q. Baig, M. Imran, W. Khalid and M. Naeem, Can. J. Chem., 2017, 95, 674-686.

7. S. Hayat and M. Imran, Appl. Math. Comput., 2014, 240, 213-228.

8. H. Wiener, J. Am. Chem. Soc., 1947, 69, 17-20.

9. I. Gutman and O. E. Polansky, "Mathematical Concepts in Organic Chemistry", Springer-Verlag, New York, 1986.

10. I. Gutman and N. Trinajstic, Chem. Phys. Lett., 1972, 17, $535-538$. 
11. B. Furtula and I. Gutman, J. Math. Chem., 2015, 53, 1184-1190.

12. W. Gao, M. K. Siddiqui, M. Imran, M. K. Jamil and M. R. Farahani, M. R. Saudi Pharma. J., 2016, 24, 258-267.

13. B. Furtula, A. Graovac and D. Vukicevic, J. Math. Chem., 2010, 48, 370-380.

14. A. T. Balaban, Chem. Phys. Lett., 1982, 89, 399-404.

15. A. T. Balaban and L. V. Quintas, J. Math. Chem., 1983, 14, 213-233.

16. P. S. Ranjini, V. Lokesha and A. Usha, Int. J. Graph. Theory, 2013, 1, 116-121.

17. M. Bača, J. Horváthová, M. Mokrišová and A. Suhányiová, Appl. Math. Comput., 2015, 251, 154-161.

18. M. Bača, J. Horváthová, M. Mokrišová, A. Semanicov $\tilde{A}_{i}$-FenovcÃ-kovÃ $i$ and A. Suhányiová, Cana. J. Chem., 2015, 93, 1-4.

19. S. Hayat and M. Imran, J. Comput. Theor. Nanosci., 2015, 12, 533-541.

20. M. Imran, M. K. Siddiqui, M. Naeem and M. A. Iqbal, Symmetry, 2018, 10, 173-183.

21. W. Gao, W. Wang and M. R. Farahani, J. Chem., 2016, 10, $1-10$.

22. I. Gutman, Croat. Chem. Acta., 2013, 86, 351-361.

23. B. Basavanagoud, W. Gao, S. Patil, V. R. Desai, K. G. Mirajkar and B. Pooja, Appl. Mathem. Nonlinear Scie., 2017, 2, 285-298.
24. D. Liu, C. Wang and S. Wang, Appl. Mathem. Nonlinear Scie., 2018, 3, 419-426.

25. J. A. Aledo, L. G. Diaz, S. Martinez and J. C. Valverde, Appl. Mathem. Nonlinear Scie., 2018, 3, 593--602.

26. M. Knor, R. Skrekovski and A. Tepeh, Appl. Mathem. Nonlinear Scie, 2018, 3, 433-446.

27. K. S. Novoselov, A. K. Geim, S. V. Morozov, D. Jiang, Y. Zhang, S. V. Dubonos, I. V. Grigorieva and A. A. Firsov, Science, 2004, 306, 666-669.

28. P. Li, R. Zhou and X. C. Zeng, Nanoscale, 2014, 6, 116126.

29. M. K. Siddiqui, M. Imran and A. Ahmad, Appl. Math. Comput., 2016, 280, 132-139.

30. M. K. Siddiqui, M. Naeem, N. A. Rahman and M. Imran, JOAM, 2016, 18, 884-892.

31. K. Chen, C. Sun, S. Song and D. Xue, Cryst. Eng. Comm., 2014, 16, 52-57.

32. B. D. Yuhas and P. Yang, J. Am. Chem. Soc., 2009, 131, 37-46.

33. J. Zhang, J. Liu, Q. Peng, X. Wang and Y. Li, Chem. Mater., 2006, 18, 867-877.

34. N. N. Greenwood and A. Earnshaw, "Chemistry of the Elements", second edition, Butterworth, UK, 1997.

35. F. A. Cotton, G. Wilkinson, C. A. Murillo,M. Bochmann, "Advanced Inorganic Chemistry", John Wiley and Sons, 1999. 
